\title{
Améloblastome : diagnostic et traitement. A propos de 26 cas
}

\section{Ameloblastoma : diagnosis and treatment. Report of 26 cas}

\author{
SOPHIA NITASSI ${ }^{1}$, MALIK BOULAADASS ${ }^{1}$, IMANE TOBI ${ }^{2}$, LAILA ESSAKALI ${ }^{1}$, MOHAMMED KZADRI $^{1}$
}

\begin{abstract}
RÉSUMÉ
L'améloblastome est une tumeur épithéliale odontogène histologiquement bénigne mais ayant un comportement malin par son pouvoir invasif et déformant local.

Ce travail porte sur une série de 26 cas colligés dans le service d'ORL et de Chirurgie maxillo-faciale de l'Hôpital des Spécialités de Rabat, entre janvier 2000 et février 2007.

L'âge moyen des patients était de 30 ans avec un sex ratio de 1. La majorité des patients n'ont consulté qu'au stade de la tuméfaction. La localisation la plus fréquente était la mandibule avec 23 cas $(88,4 \%)$. Sur le plan thérapeutique : 4 ont bénéficié d'un traitement conservateur et 22 d'un traitement radical. On a noté un taux de récidive de $23 \%$ (6/26 cas) ce qui correspond aux données de la littérature.

Ce sujet a fortement suscité notre intérêt du fait de sa fréquence relativement élevée dans notre pays comparée aux données de la littérature, de la difficulté de prise en charge thérapeutique en raison du pouvoir récidivant et mutilant de ces tumeurs, et également du rôle du chirurgien dentiste dans le dépistage précoce.

(Med Buccale Chir Buccale 2009 ; 15: 93-100).
\end{abstract}

mots clés: améloblastome, diagnostic, traitement

\section{SUMMARY}

Ameloblastoma is an epithelial odontogenic tumour; harmless of part its histology but clever of part its invasif power and local distorting.

This study concerned a series of 26 colligated cases in ORL and maxillofacial surgery services at Hospital of the Specialities of Rabat between January 2000 and February 2007.

The medium age of our patients was 30 years with a sex ratio of 1 . The majority of the patients saw only at the stage of tumefaction. The most frequent location was in mandible with 23 cases (88,4\%).

On therapeutic plan, 4 benefited from a conservative treatment and 22 of a radical treatment. It noted a rate of repetition of $23 \%$ (6/26 cases) which joins the data of literature.

This subject hard provoked our interest due to its relating frequency in our country contrary to foreign data and, especially, difficulty of was taken care therapeutics seen the committing a second offence and wounding power of these tumours and also of the role of the surgeon dentist in the early detection. (Med Buccale Chir Buccale 2009 ; 15: 93-100).

key words: ameloblastoma, diagnostic, treatment médecine

buccale

chirurgie

buccale

VOL. $15, \mathrm{~N}^{\circ} 2$

2009

page 93

1. Service d'Otorhinolaryngologie et de Chirurgie maxillo-faciale Hôpital des Spécialités Rabat

2. Docteur en Médecine dentaire en exercice libéral

Demande de tirés à part:

Sophia Nitassi Résidence Ahssan Dar avenue Hassan II ImmB Appt33 Agdal Rabat sophian79@hotmail.com 
L'améloblastome est un néoplasme épithélial développé à partir des cellules dento-formatrices sans composante mésenchymateuse. II représente $10 \%$ des tumeurs odontogènes et $1 \%$ des tumeurs des tissus osseux des maxillaires [1]. II se traduit longtemps par des manifestations cliniques frustes avant l'apparition de la tuméfaction. Ces cas vont permettre d'étudier les différents aspects épidémiologiques, cliniques et thérapeutiques de cette tumeur.

\section{MATÉRIEL ET MÉTHODES}

L'étude a porté sur une série de 26 cas d'améloblastomes se rapportant aux dossiers exploitables des patients traités dans le service d'ORL et de Chirurgie maxillo-faciale de l'Hôpital des Spécialités de Rabat sur une période de 6 ans s'étalant entre

médecine buccale chirurgie buccale

VOL. $15, \mathrm{~N}^{\circ} 2$ 2009 page 94 janvier 2000 et février 2007. On n'a retenu que les améloblastomes endo-osseux.

\section{RÉSULTATS}

Le sex ratio est de 1, avec 13 cas de sexe masculin et 13 de sexe féminin. L'âge moyen est de 30 ans, avec des âges extrêmes de 16 ans et 70 ans. Dans 23 cas, les patients étaient de race blanche d'origine marocaine et dans 3 de race noire d'origine mauritanienne. Les localisations mandi-
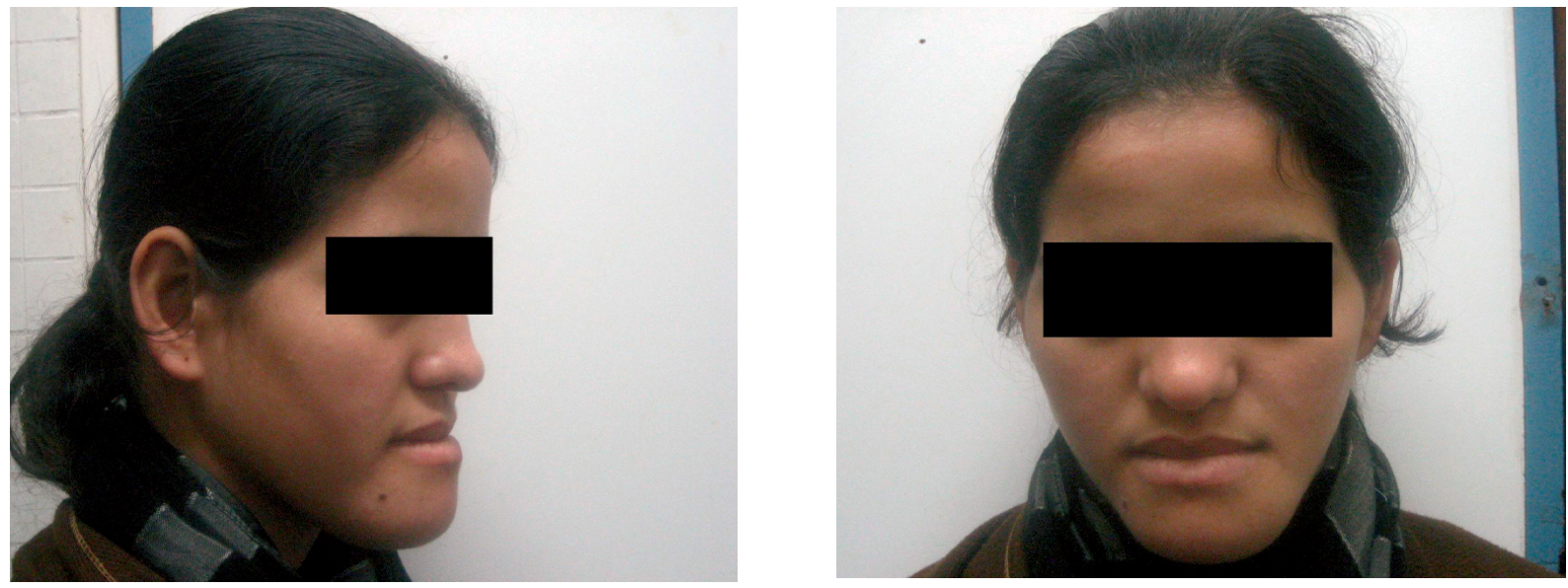

Figure 1 : Un cas d'améloblastome symphysaire : vue de face et de profil. A symphisis ameloblastoma case: view of face and profile.

bulaires représentaient 23 cas $(88,4 \%)$; les localisations maxillaires 1 cas et, dans 2 cas, il y avait une double localisation. Dans les localisations mandibulaires, la tumeur touchait toute une hémi-mandibule dans 9 cas, la mandibule dans sa totalité dans 1 cas ; pour la localisation maxillaire, l'améloblastome intéressait la tubérosité. Dans les deux cas avec double localisation, l'un avait une localisation maxillaire primitive (sinus maxillaire, fosse infra-temporale et base du crâne) avec envahissement secondaire du ramus, pour l'autre le point de départ était indéterminé car il touchait toute l'hémimandibule gauche, la fosse ptérygo-maxillaire et le sinus maxillaire.

Cliniquement, la tumeur se traduisait le plus souvent par une tuméfaction dure et ferme ; sa localisation était : mentonnière dans 2 cas, massétérine dans 1 cas, génienne dans 1 cas également, en regard du sinus maxillaire droit dans 1 cas et en regard de la tubérosité maxillaire dans 1 cas (Fig. 1). II existait une douleur chez 5 patients. Les signes dentaires étaient d'importance variable, parfois ils constituaient le premier symptôme : mobilités dentaires (2 cas), déplacements dentaires ( 1 cas), troubles de l'articulé dentaire (1 cas), inclusion dentaire (2 cas), absence de dent (8 cas). Un patient a eu un traitement orthodontique pour des déplacements dentaires induits par l'améloblastome sousjacent. D'autres signes pouvaient être associés : présence d'une lésion gingivale ulcéro-bourgeonnante (4 cas), fistule (2 cas), gingivorragies (1 cas), 


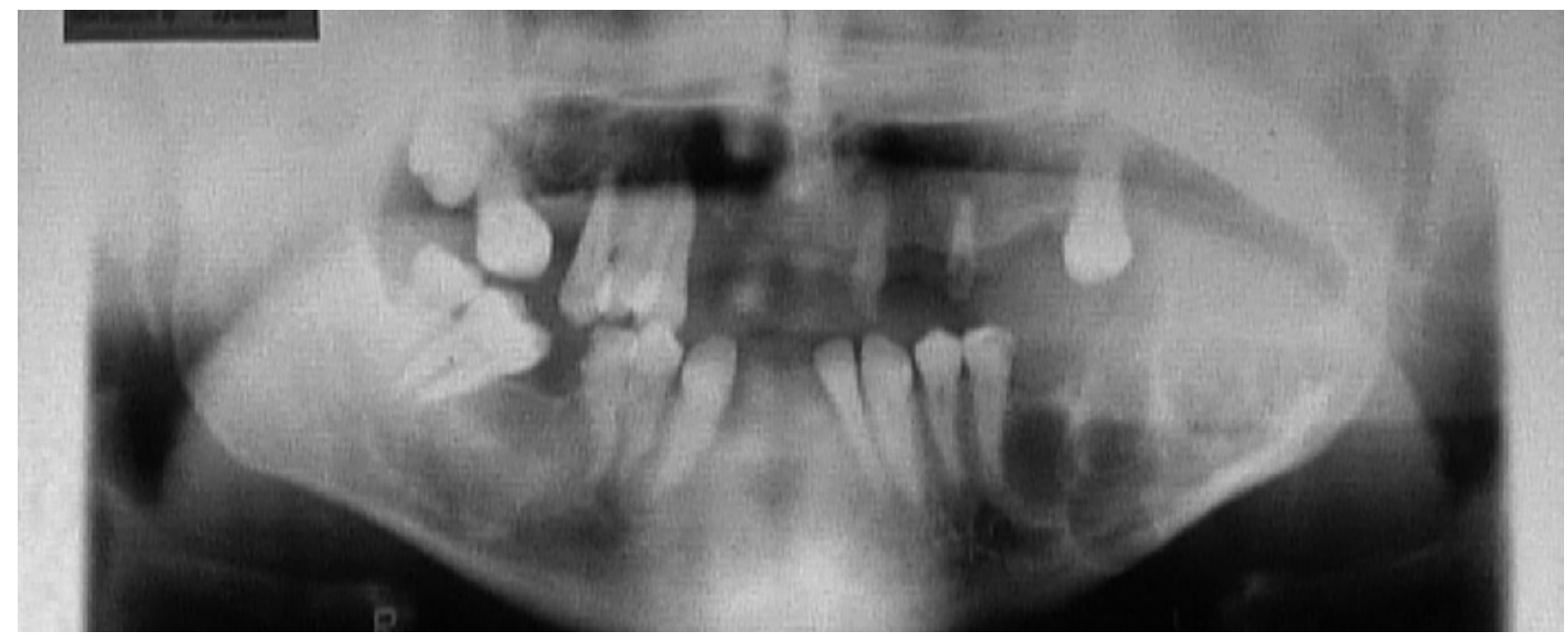

Figure 2 : Radiographie panoramique montrant l'aspect polygéodique de l'améloblastome intéressant la branche horizontale et le ramus mandibulaire.

Panoramic X-ray showing the ameloblastoma polygeodic aspect

limitation de l'ouverture buccale (3 cas), algies faciales (2 cas). Une rhinorhée purulente associée à une exophtalmie avec diplopie a été observée dans 1 cas. La découverte a été fortuite chez un patient lors d'un bilan radiologique.

Sur le plan radiologique, un orthopantomogramme a été réalisé chez 21 patients, une TDM chez 15 patients et une IRM chez 1 patient. Dans 11 cas, l'image était polygéodique, dans 6 cas monogéodique (Fig. 2 et 3). Dans 3 cas, il s'agissait d'une lésion ostéolytique avec dans 2 cas la soufflure des corticales mandibulaires et dans 1 cas un envahissement des parties molles.

Une volumineuse et nette masse lytique dans le ramus a été mise en évidence sur la TDM multibarette (3D) dans 1 cas (Fig. 4). Dans 1 cas, il s'agissait d'une ostéolyse diffuse.

Dans tous les cas, le diagnostic a été confirmé par l'étude anatomopathologique en microscopie optique (Fig. 5).

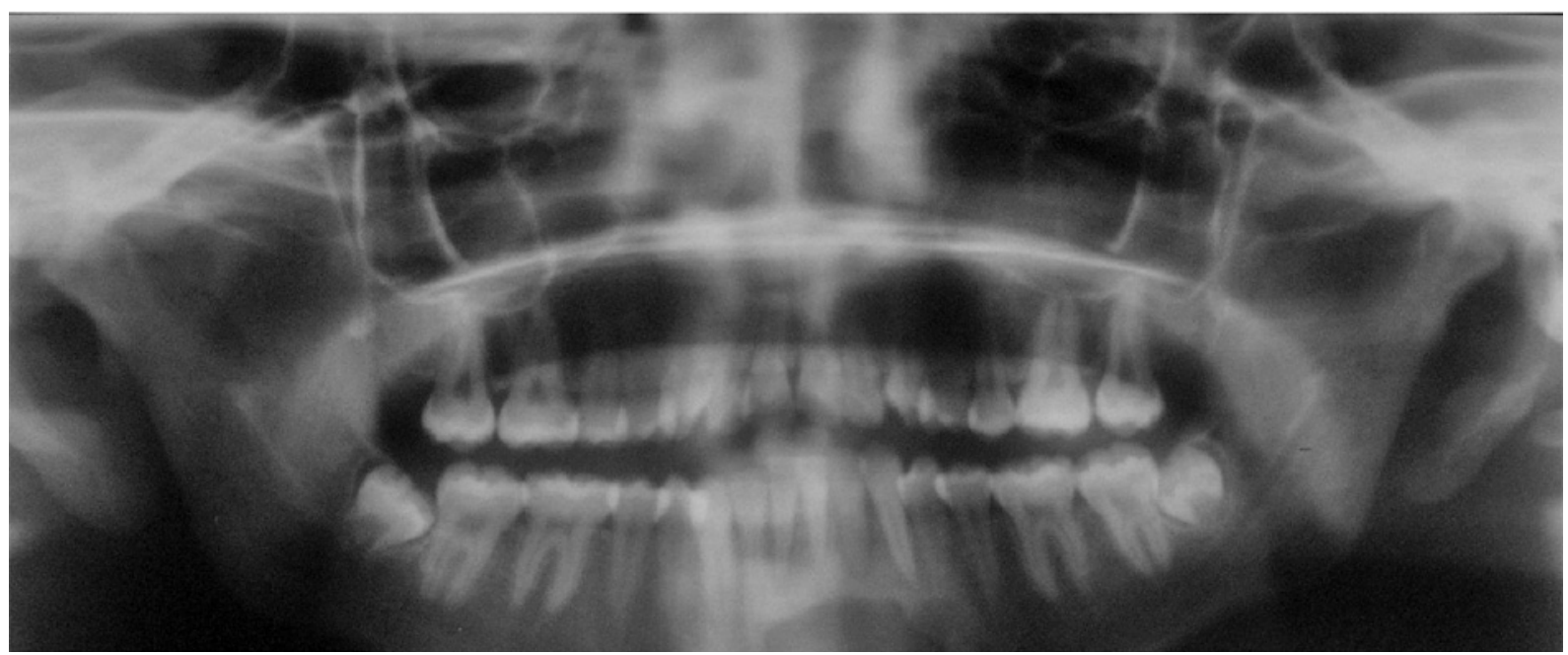

Figure 3 : Orthopantomogramme montrant l'image monogéodique d'un améloblastome symphysaire.

Panoramic X-ray showing the ameloblastoma monogeodic aspect. 


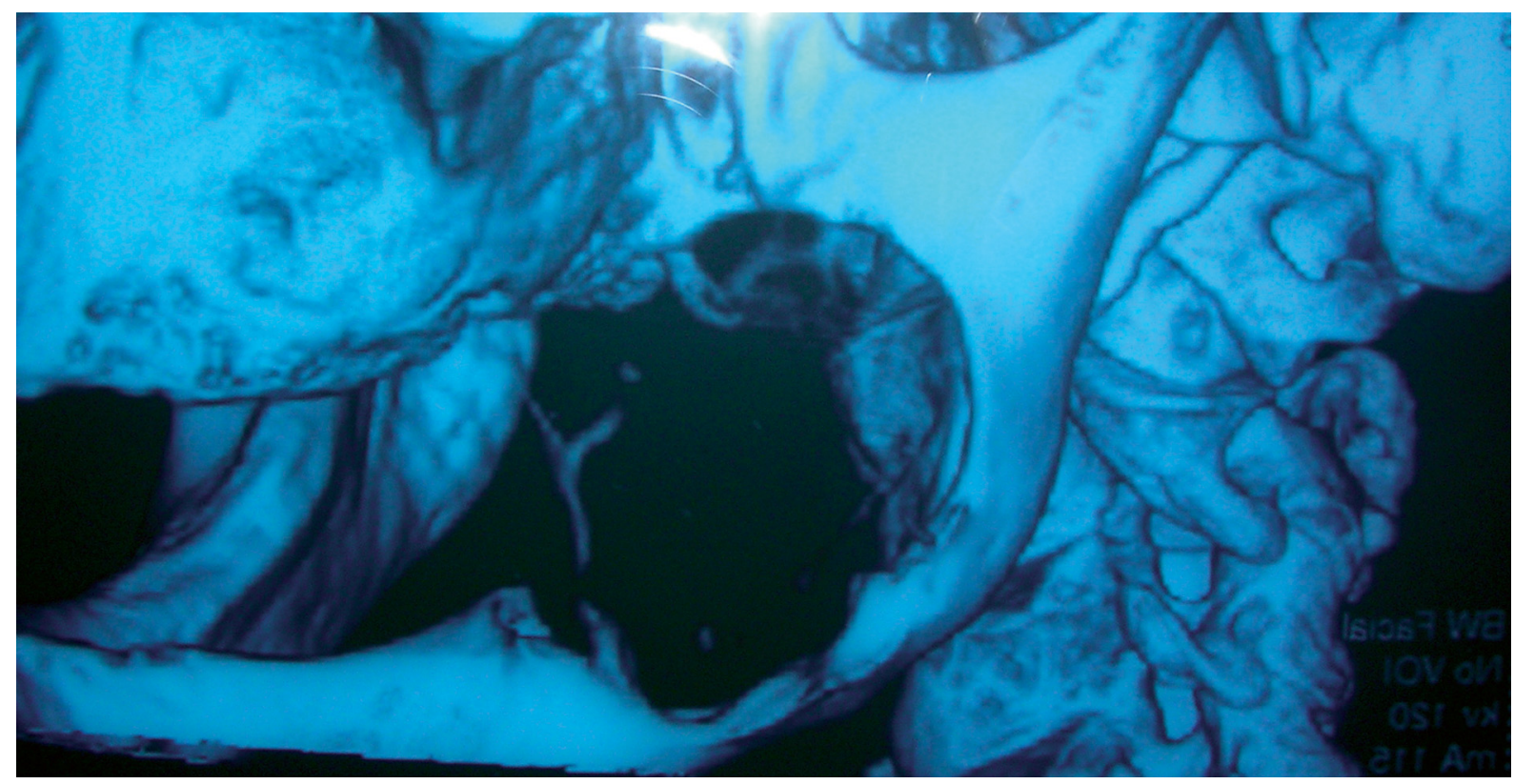

Figure 4 : Améloblastome du ramus mandibulaire en TDM 3D.vue de profil.

médecine Picture of a ramus localisation ameloblastoma at a 3D tomodensitometry in a profile view.

buccale

chirurgie

buccale

VOL. $15, \mathrm{~N}^{\circ} 2$

2009

page 96

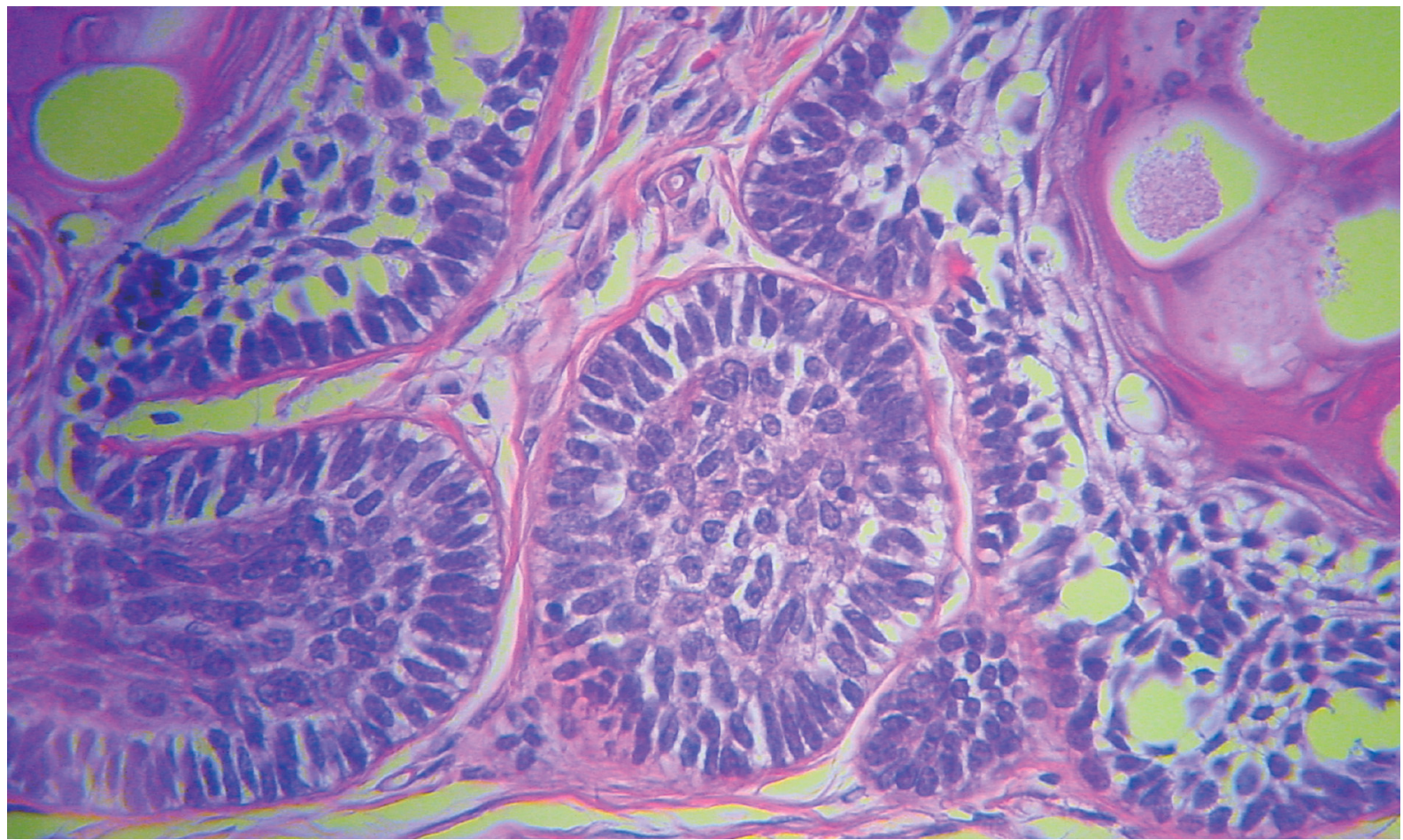

Figure 5 : Follicule améloblastique tapissé par un épithélium cylindrique palissadique en périphérie et étoilé au centre, entouré par une condensation fibro-hyaline (HES, X 400).

Ameloblastic follicle wallpapered by a cylindrical epithelium palisadic in periphery and starry in the centre encircled by a fibro-hyaline condensation (HES, X 400). 
Tous nos patients ont été opérés. Le traitement conservateur a été réalisé chez 6 patients : il a consisté en un curetage appuyé dans 6 cas et, dans 2 cas, ce curetage a été réalisé à 2 reprises. $\|$ a été associé à une énucléation dans 1 cas. Dans 1 cas, il a consisté en une "kystectomie » avec marsupialisation. Un traitement radical a été réalisé chez 22 patients, pour 4 cas après un traitement conservateur. II a consisté en une hémi-mandibulectomie dans 18 cas. Une exérèse tumorale simple au maxillaire a été réalisée dans 1 cas et, dans l'autre cas, il a consisté en une hémi-maxillectomie droite avec exérèse du ramus mandibulaire droit.

La réhabilitation de la perte de substance osseuse a été réalisée chez 10 patients : par greffon iliaque dans 4 cas ; par mini plaques et vis dans 3 cas, par greffon du péroné dans 2 cas. Une distraction osseuse pour gain d'espace a été réalisée chez de 2 de ces 10 patients.

II y a eu une récidive dans 6 cas : 5 localisations mandibulaires et 1 localisation maxillaire. II s'agissait de 2 épisodes de récidives dans 2 cas et de 4 récidives dans 1 cas ; 5 des 6 récidives ont été observées après un traitement conservateur.

\section{DISCUSSION}

Relativement fréquent parmi les tumeurs odontogéniques mais rare si l'on considère des tumeurs et kystes des maxillaires, l'améloblastome ne représente que $1 \%$ des tumeurs osseuses [1] et 25,2 à $58,6 \%$ des tumeurs odontogéniques bénignes selon les auteurs [2-4]. La répartition géographique est variable selon le pays d'origine et l'ethnie, l'améloblastome n'a aucun lien avec la région d'habitation, ni avec le climat mais la fréquence est nettement plus élevée dans la population noire africaine; elle peut atteindre jusqu'à $80,1 \%$ dans certaines séries ${ }^{[5]}$. II survient essentiellement entre la 3ème et 4ème décade avec une légère prédisposition pour les sujets de 20 à 35 ans ${ }^{[6,7]}$. Dans notre série, la moyenne d'âge était de 30 ans avec des extrêmes de 16 et de 70 ans. Il affecte autant le sexe féminin que masculin $[6,8,9]$.

L'améloblastome intéresse principalement la mandibule (80\% des cas). La région angulaire repré- sente la localisation la plus fréquente, avec extension vers la branche horizontale (70 \%), puis viennent ensuite les régions parasymphysaires (20\%) et symphysaire (10\%). La tubérosité constitue la localisation maxillaire préferentielle.

Dans cette série, si l'on exclut les 2 cas ayant une double localisation, la localisation mandibulaire représente $87,5 \%$ (23 cas sur 24 ).

L'améloblastome se caractérise par une latence prolongée, sa découverte étant souvent fortuite lors d'un examen radiologique pour des soins dentaires. Dans cette série, les patients ont consulté au stade de la tuméfaction en raison de la rareté des soins dentaires dans cette population ; retard encore accru pour les patients mauritaniens par les formalités de rapatriement [10-12]. La tuméfaction est en général dure, ferme, de consistance osseuse, sans signes inflammatoires en regard. Dans cette série, les améloblastomes douloureux ( 5 cas) étaient associés à la tuméfaction.

Les signes dentaires le plus souvent retrouvés dans la littérature sont les suivants : mobilités dentaires, déplacements dentaires, anomalies d'évolution des dents dans les régions prémolaire et molaire, retard de cicatrisation alvéolaire après avulsions intempestives... Dans tous les cas, deux signes négatifs sont à souligner : l'absence d'adénopathies satellites et l'absence d'altération de la sensibilité cutanéo-muqueuse.

L'imagerie médicale constitue un examen essentiel pour établir le diagnostic d'améloblastome. Elle fait appel aux examens conventionnels, voire à la TDM ou à l'IRM qui permettent une étude plus approfondie. L'image radiologique n'est pas univoque et aucune n'est spécifique; elle permet seulement d'orienter le diagnostic. Ce dernier repose sur un faisceau d'arguments : cliniques, épidémiologiques et surtout histologiques. A la mandibule on peut observer deux types d'images : des images multiloculaires $(2 / 3$ des cas) séparées ou des images uniloculaires (1/3 des cas) [13-15]. Au maxillaire, on distingue trois types d'images. A côté des images uni- ou multiloculaires observées à la mandibule, l'améloblastome peut entrainer une opacité sinusienne (image la plus évocatrice) : c'est le cas de l'observation $n^{\circ} 23{ }^{[12]}$. médecine

buccale

chirurgie

buccale

VOL. $15, \mathrm{~N}^{\circ} 2$ 2009

page 97 
médecine

buccale

chirurgie

buccale

VOL. $15, \mathrm{~N}^{\circ} 2$ 2009

page 98
L'orthopantomogramme sous-estime souvent l'importance d'une lésion tumorale surtout dans la localisation maxillaire et peut parfois ne pas révéler une lésion évidente à la TDM. Devant une symptomatologie traînante, il ne faut pas s'arrêter à la pratique d'un orthopantomogramme mais demander une TDM. A la TDM, il s'agit généralement d'une tumeur extensive entourée d'une coque osseuse, avec des limites irrégulières comportant des zones kystiques hypodenses et des zones isodenses solides avec prise de contraste de la composante osseuse après injection. De plus, la TDM 3D permet de montrer avec précision les limites de la tumeur et ses rapports avec le canal dentaire inférieur ${ }^{[16]}$.

L'IRM est supérieure à la radiographie standard et à la TDM dans le sens où elle permet une étude de la ou des composante(s) tumorale(s), des caractéristiques des parois « kystiques » et de la nature de la partie fluide. Cependant l'analyse spatiale tumorale est moins bonne avec l'IRM qu'avec la TDM hélicoïdale [17]. L'imagerie médicale est un élément essentiel pour établir le diagnostic et le suivi d'améloblastome.

L'améloblastome est une tumeur bénigne des maxillaires qui requiert, comme toutes les tumeurs odontogènes, un traitement chirurgical. Le traitement chirurgical, conservateur ou radical, varie et diffère selon les cas ; la décision thérapeutique doit prendre en considération plusieurs facteurs dont l'âge du patient, la localisation anatomique de la lésion, son extension, l'aspect radiologique, le potentiel évolutif et la probabilité d'un suivi régulier du patient. Malgré sa bénignité structurale, l'améloblastome présente une agressivité qui le rapproche des tumeurs malignes. En raison de son agressivité et de sa tendance à la récidive, l'améloblastome est rarement traité comme une tumeur bénigne : on a tendance à pratiquer une exérèse large pour empêcher ou essayer de limiter les récidives sans vraiment prendre en considération les séquelles qui en découlent [18]. On dispose de deux moyens : le traitement chirurgical conservateur symbolisé par la marsupialisation, l'énucléation et le curetage, et le traitement radical avec résection osseuse passant en tissu sain. Le choix est difficile car la tendance à la récidive des améloblastomes influence beaucoup l'attitude thérapeutique. Des taux de récidive élevés (45 à $90 \%$ ) ont été rapportés après un traitement conservateur. Cependant, ces nouvelles lésions sont fréquemment de plus petit volume et peuvent être traitées plus facilement que la lésion initiale. Ainsi la récidive n'est pas strictement synonyme d'échec thérapeutique. A l'opposé plusieurs auteurs préconisent d'emblée une résection large avec une marge de sécurité en périphérie [19]. Le traitement radical est d'emblée indiqué lorsque la lésion est polygéodique, le rebord basilaire envahi et dans la localisation maxillaire où l'extension guide la résection [19, 20]. Une étude réalisée par Ghandi et coll. a retrouvé $80 \%$ de récidive après énucléation et curetage et moins de $50 \%$ après traitement radical sur 50 cas étudiés [21]. Lau et Samman ont réalisé une étude pour déterminer le taux de récidive le plus bas après différents types de traitements de l'améloblastome unikystique : ils ont rapporté $3,9 \%$ de récidive après résection, $30,5 \%$ après énucléation seule, $16 \%$ après énucléation + application d'une solution de Carnoy, et $18 \%$ après marsupialisation avec ou sans autre traitement associé [22]. Une autre étude réalisée par Payam Farzad sur 8 cas d'améloblastome (7 mandibulaires et 1 maxillaire) : 4 traités par énucléation ou curetage, 1 cas par résection non interruptrice et 2 cas par hémimandibulectomie. On a noté 5 cas de récidive dont 3 avaient eu un traitement conservateur, 1 cas une résection non interruptrice. Le cas du maxillaire a subi une résection passant en zone saine et n'a pas récidivé [23].

Ainsi, la récidive est plus importante après un traitement conservateur que radical. D'ailleurs, la majorité des auteurs ont adopté le traitement radical car il est le seul permettant d'éviter les récidives. Hormis dans quelques cas, la récidive et les gestes chirurgicaux itératifs sont au final plus mutilants que le traitement radical; de plus, le risque de transformation maligne devient non négligeable [24]. Par contre, Bataille et coll. considèrent que si l'indication de la méthode conservatrice est bien posée et si la chirurgie bien menée, le traitement conservateur garde sa place même si elle n'est plus la première dans le traitement des améloblastomes [19-25]. Ce traitement 
respecte la continuité mandibulaire et, si possible, le pédicule vasculo-nerveux dentaire inférieur, les corticales laminées, les parties molles et les organes dentaires. II a permis à un nombre non négligeable de patients de guérir de manière simple avec des séquelles morphologiques ou fonctionnelles modérées. Chez l'enfant, le traitement conservateur est plus facilement indiqué du fait des impératifs de la croissance osseuse mais seulement dans le cas où un suivi régulier correct peut être instauré. Les 6 cas d'améloblastomes chez l'enfant traités par Takahaschi ont montré des résultats satisfaisants et aucune déformation osseuse [16].

Le risque de récidive est maximum dans les trois premières années mais la récidive peut survenir beaucoup plus tard, 15 à 30 ans après. Elle se traduit par la reprise clinique et radiologique du processus tumoral. C'est dire la nécessité d'une surveillance clinique et radiologique prolongée. Le rythme est fonction de la qualité de ré-ossification, variant entre 3 et 8 mois dans un premier temps, et la durée du suivi établie en fonction du type de la lésion initiale [15, 20, 21].

Dans notre série, on a noté également 6 cas (23\%) de récidive sur une série de 26 patients dont 1 cas maxillaire pour 5 cas mandibulaires. Tous ont eu un traitement conservateur.

Le chirurgien dentiste peut intervenir aussi bien par sa collaboration à la prévention, au dépistage précoce devant une mobilité dentaire anormale il faut penser à faire une exploration radiologique et ne pas toujours se contenter du cliché rétroalvéolaire et également dans l'incitation du patient à s'adresser à un centre spécialisé suite à la découverte de la moindre anomalie pour bénéficier d'un traitement précoce limitant la mutilation que cause une chirurgie radicale comme c'est le cas de notre patiente.

\section{CONCLUSION}

L'améloblastome est une tumeur invasive qui, malgré son caractère bénin, offre l'exemple d'une tumeur d'une singulière agressivité, caractérisée surtout par sa tendance à la récidive. II demande de ce fait un diagnostic précis et une prise en charge appropriée avec un suivi à long terme pour détecter toute récidive.

Le traitement constitue un challenge pour le chirurgien : il doit traiter le patient avec un risque faible de récidive tout en lui assurant un résultat esthétique et fonctionnel optimal, d'où l'éternelle discussion lors du choix de l'option thérapeutique : traitement radical ou traitement conservateur?

Le chirurgien dentiste intervient dans le dépistage précoce et également dans le suivi postopératoire et la réhabilitation prothétique.

\section{RÉFÉRENCES}

1 - Chomette G, Auriol M. Histopathologie buccale cervico-faciale (pp.51-57) Masson, Paris, 1986.

2 - Olgac V, Koseoglu BG, Aksakalli N. Odontogenic tumours in Istambul: 527 cases. Br J Oral Maxillofac Surg $2006 ; 44$ : 386-8.

3 - Fernandez AM. Odontogenic tumours: a study of 340 cases in brazilian population. J Oral Pathol Med 2005 ; $34: 583-7$.

4 - Lu Y, Xuan M, Takata T, Wang C, He Z, Zhou Z, Mock D, Nikai $\mathrm{H}$. Odontogenic tumour: a demographic study of 759 cases in a Chinese population. Oral Surg Oral Pathol Oral Radiol Endod 1998 ; 86 : 707-14.

5 - Simon EN, Merkx MA, Vuhahula E, Ngassapa D, Stoelinga PJ. A 4 - year prospective study on epidemiology and clinicopathological presentation of odon- togenic tumours in Tanzania. Oral surg. Oral Pathol Oral Radiol Endod 2005 ; 99 : 598-602.

6 - Ladeinde $A L$, Ogunlewe $M O$, Bamgbose $B O$, Adeyemo WL, Ajayi OF, Arotiba GT, Akinwande JA. Ameloblastoma analysis of 207 cases in Nigerian teaching hospital. Quintessence Int 2006 ; 37 : 69-74.

7 - Arotiba GT, Ladeinde AL, Arotiba JT, Ajike SO, Ugboko VI, Ajayi OF. Ameloblastoma in Nigerian children and adolescents; a review of 79 cases. J Oral Maxillofac Surg 2005 ; 63 : 747-51.

8 - Ruhin B, Guilbert F, Fouret P, Ghoul S, Berdal A, Bertrand JC. Tumeurs des maxillaires ; améloblastome : données actuelles et perspectives. Rev Stomatol Chir Maxillofac 2005 ; 106 : 1 s64. médecine

buccale

chirurgie

buccale

VOL. $15, \mathrm{~N}^{\circ} 2$ 2009

page 99 
9 - Regezi A, Sciubba J. Oral pathology: clinical pathologic correlations (pp. 337-48). Saunders Company, StLouis (USA), 1989.

10 - Favre-Dauvergne E, Auriol M, Le Charpentier Y. Tumeurs odontogeniques. Encycl Med Chir Paris. 22-062-F-10, 1995.

11 - Dechaume M. Precis de Stomatologie (pp 560-5). Masson, Paris, 1966.

12 - Benaazza D. L'améloblastome : étude à propos de 32 cas. Thèse de Médecine dentaire n 95, Rabat, 2005.

13 - Barthe M, Daudibertieres L. Classification radiologique des tumeurs bénignes ostéo-mandibulaires. Rev Odontostomatol. 1994 ; 23 : 45-56.

14 - Bennani A. Les kystes et les tumeurs kystiques bénignes de la mandibule : place du bilan radiologique dans la démarche diagnostique. Thèse de Médecine dentaire, Rabat, 2006 http://hdl.handle.net/123456789/266.

15 - Benhalima A, Lazrak A. Diagnostic des tumeurs et pseudo-tumeurs mandibulaires. 55-61; 134.

16 - Chala S. Les améloblastomes de la mandibule a propos de 16 cas. Thèse de Médecine dentaire $n^{\circ} 33$, Rabat, 2000.

médecine buccale chirurgie buccale

VOL. $15, \mathrm{~N}^{\circ} 2$ 2009
18 - Olazoji H, Enwere O. Treatment of ameloblastoma - a review. Niger J Med 2003; 12: 7-11.

19 - Ruhin B, Guilbert F, Bertrand J. Traitement des kystes, tumeurs et pseudotumeurs bénignes des maxillaires. Encycl Med Chir. Paris, 22-062-K-10, 2005.

20 - Olaitan AA, Arole G, Adekeye EO. Recurrent ameloblastoma of the jaws: a follow-up study. Int Journal Oral Maxillofac Surg $1998 ; 27:$ 456-60.

21 - Ghandhi D, Ayoub AF, Pogrel MA, MacDonald G, Brocklebank LM, Moos KF. Ameloblastoma: surgeon's dilemma. J Oral Maxillofac Surg 2006 ; 64 : 1010-4.

22 - Lau SL, Samman N. Reccurence related to treatment modalities of unicystic ameloblastoma: a systematic review. Int J Oral Maxillofac Surg 2006 ; 35 : 681-90. Epub 2006 jun 16.

23 - Payam F. Ameloblastoma of the jaws. Institute of odontology, Karolinska Institute, Huddinge.

24 - Benhalima H, Rafi H, Slaoui S, Kzadri M. L'améloblastome mandibulaire : problèmes thérapeutiques. Med Maghreb. 1992, 34.

25 - Bataille R, Schumann CL, Seghir M. A propos de plusieurs cas d'améloblastomes mandibulaires primitives ou recidivées traits par curettage chirurgical. Rev Stomatol Chir Maxillofac $1974 ;$; 75 : 33-6. 